\title{
Sécheresse, écologie et lutte contre Glossina tachinoides dans la région du Bas-Chari
}

\author{
par J. GRUVEL (*)
}

\begin{abstract}
RESUME
La sécheresse intense qui s'installe en Afrique sahélienne depuis quelques années exerce ćgalement son influence au niveau des galeries ripicoles de la vallée du Chari. Elle modifie très sensiblement:

- les conditions écologiques vis-à-vis de $G$. tachinoides qui voit ses populations se réduire en limitant leur présence aux seuls rares endroits épargnés par le dessèchement;

- les conditions d'application de l'insecticide au cours de l'exécution de la campagne de lutte menée actuellement en aval de N'Djamena.
\end{abstract}

L'Afrique sahélienne connaît depuis plusieurs années des conditions de sécheresse exceptionnelle qui s'étendent à la région de N'Djamena et notamment à la vallée du basChari.

Les deux grands facteurs de l'économie nationale, agriculture et élevage, sont durement touchés par ces modifications climatiques qui, en réduisant l'hydrographie de la région, entraînent un flétrissement de la végétation, non seulement de savane, mais également des bords du fleuve. En ces endroits, il modifie les biotopes de Glossina tachinoides et entraîne des variations de sa distribution et de sa densité.

Cette action résulte:

$\rightarrow$ de la diminution des pluies annuelles locales dont la répartition, trop étalée dans le temps, ne permet pas une saturation normale des terrains;

- de la réduction des zones d'inondation par le fleuve et ses défluents dont la baisse des niveaux, maximal et minimal, résulte de l'insuffisance et du mauvais étalement des pluies sur les hautes vallées du Chari et du Logone.

(*) I.E.M.V.T., Laboratoire de Farcha, B.P. 433, N'Djamena, Tchad.
Cette note a pour but de mettre en évidence l'influence de cette période de sécheresse sur l'écologie de $G$. tachinoides, plus particulièrement dans la vallée du bas-Chari, et d'en dégager quelques remarques sur l'application des méthodes de lutte par pulvérisation d'insecticide.

\section{SECHERESSE ET ECOLOGIE}

DE G. TACHINOIDES

(Fig. 1 - Répartition - Carte)

On sait que la répartition de Glossina tachinoides $\mathrm{W}$. dans la portion tchadienne et camerounaise du bassin du lac Tchad est liée au réseau hydrographique constitué par le Logone, le Chari, leurs affluents et leurs défluents dont l'El Beid pour le premier, le Bahr Erguig, le Serbewel et le Taf-taf pour le second, sont les plus importants.

- Les débordements annuels des fleuves alimentent normalement ces défluents qui conservent alors une grande partie de leur eau après la décrue. Tous ces cours d'eau, ainsi que quelques mares résiduelles, sont bordés naturellement par une végétation ripicole qui cons- 


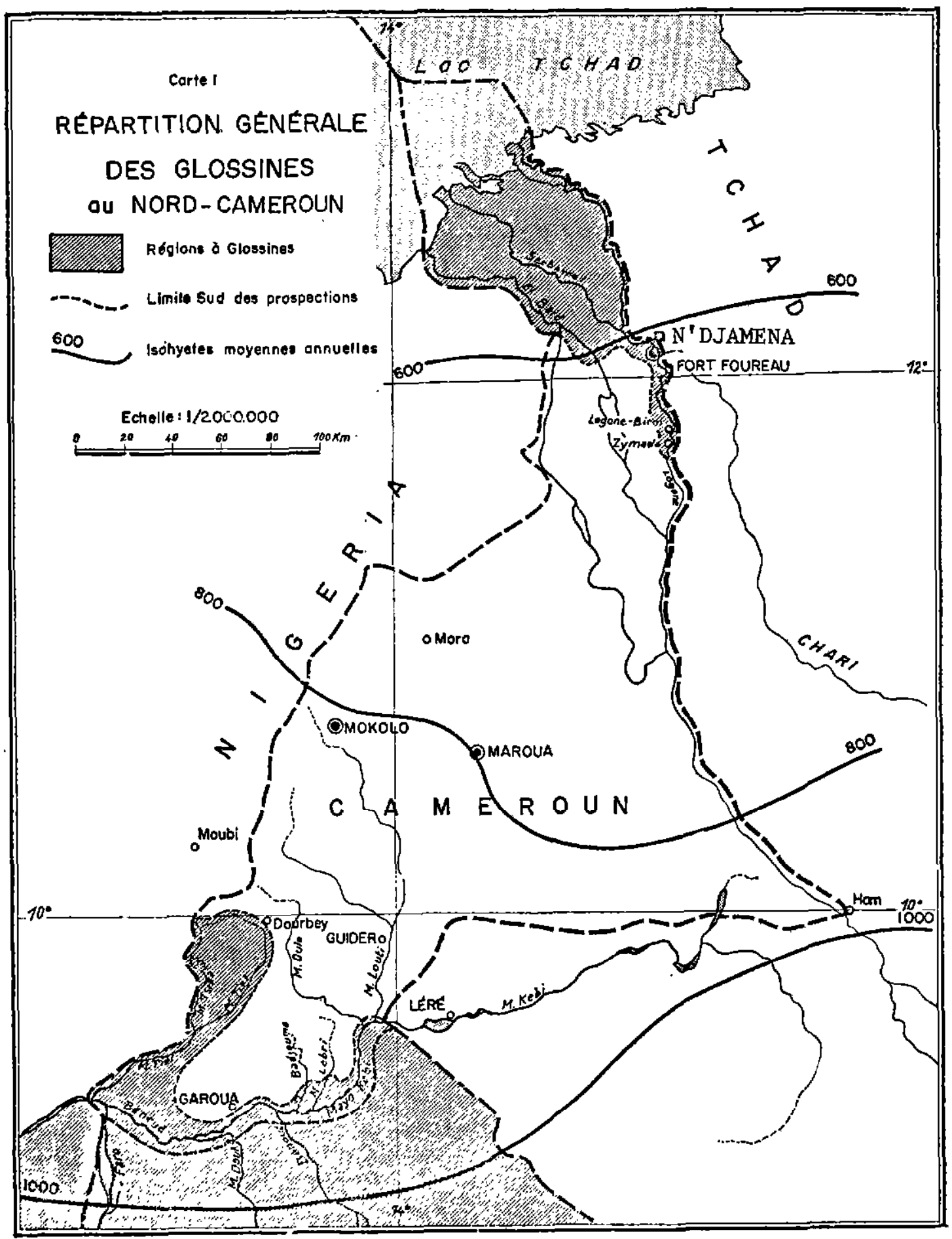

titue l'habitat typique de $G$. tachinoides et $3\left(^{*}\right)$.

- Ces habitats sont inégalement occupés par les glossines au cours de l'année; suivant les

(*) Les numéros en italique renvoient à la bibliographie; ceux entre parenthèses aux zones de végétation.
(**) Nous remercions vivement les chercheurs de la Section d'Hydrologie qui nous ont aimablement communiqué ces renseignements. 
TABLEAU $N^{\circ}$ I. -Variations des niveaux du Chari au cours des dix dernières années.

\begin{tabular}{|c|c|c|c|c|}
\hline Périades & $\begin{array}{c}\text { variations de } \\
\text { M. }\end{array}$ & $\begin{array}{c}\text { hauteurs du } \mathrm{C} \\
\mathrm{m} .\end{array}$ & $\begin{array}{l}\text { lar } 1 \text { (mètre) } \\
\text { Amplitude }\end{array}$ & $0 b s$ ervations \\
\hline $\begin{array}{l}\text { Moyennes de } \\
1960 \text { à } 1970\end{array}$ & 7,23 & 0,92 & 6,31 & $\begin{array}{l}M: 2 B \text { octobre; m : } 2 B \text { avrıl } \\
\text { (niveau inférieur à } 4,08 \text { mètres } \\
\text { pendant } 8 \text { mols) }\end{array}$ \\
\hline Annëe 1970 & 8,17 & 1,19 & 6,98 & M : 25 octobre; m : 27 avril. \\
\hline Année 1971 & 7,50 & 1,12 & 6,38 & $\begin{array}{l}\text { M : } 16 \text { octobre; m : } 28 \text { mai } \\
\text { (m:étal depuis } 14 \text { mai) }\end{array}$ \\
\hline Annëe 1972 & 4,29 & 1,00 & 3,29 & $\begin{array}{l}\text { M : en palier du } 8 \text { septembre } \\
\text { au } 11 \text { novembre. }\end{array}$ \\
\hline Arnnée 1973 & 5,55 & 0,63 & 4,92 & $\begin{array}{l}\text { m:en palier du } 12 \text { au } 30 \text { avril } \\
\text { M:fin octobre. }\end{array}$ \\
\hline
\end{tabular}

$M=$ maxımum; $\mathrm{m}=$ minimum.

sont données par les maximums et minimums, pour les dernières années, dans le tableau I.

- On remarque que, à partir de 1970 , les valeurs caractéristiques accusent une baisse sensible, particulièrement remarquable en 1972 et 1973; le niveau minimal ayant été exceptionnellement bas cette dernière année. De plus, les maximums et les minimums qui se manifestent habituellement d'une manière très franche, à des intervalles de 6 mois, se trouvent maintenant émoussés et montrent même un étalement tout à fait anormal. Celui des maximums de 1972 est particulièrement saisissant.

- Les conséquences générales de ces baisses de niveau se font sentir nettement depuis la fin de l'année 1972 dans les défluents du Logone et du Chari :

- en amont de N'Djamena, l'El Beid et le Bahr Erguig, alimentés normalement, respectivement, par les débordements du Logone par les yaérés du nord-Cameroun et par ceux du Chari aux environs de Miltou, se sont trouvés dès le début de la dernière saison chaude, le premier totalement et le second partiellement à sec;

- plus localement, en aval de N'Djamena (vallée du bas-Chari), le Serbewel et le Taf-taf ont été totalement asséchés dès la fin du mois de mars 1973. De nombreuses mares intérieures ont, pour la première fois depuis très longtemps, été elles aussi entièrement privées d'eau.

Les populations de $G$. tachinoides se maintiennent dans des groupements végétaux ripicoles dont la composition, la distribution et la persistance dépendent de la durée des inondations qu'ils subissent 4 . Elles suivent ou pré- cèdent annuellement la descente ou la montée des eaux et se trouvent localisées entre les portions des galeries végétales ripicoles, ou trop arides de l'intérieur, ou inondées des parties basses.

- En année normale, trois zones de végétation caractéristiques peuvent ainsi être distinguées (fig. 2, p. 454):

- La zone (1), peu ou pas inondée, où les glossines sont présentes de fin août à début décembre;

- La zone (2), inondable près de 3 mois, est occupée par les mouches à la descente et à la montée des eaux : de novembre à maṛs puis de fin juillet à début septembre;

- La zone (3) inondée plus de 6 mois en moyenne chaque année, est habitée par les tsé-tsé de janvier à fin juillet. Les Morelia senegalensis forment les gîtes de cette zone qui se prolonge, vers les eaux libres, par des massifs de Mimosa pigra dont la plus grande surface est normalement immergée.

- Le tableau II rend compte des déplacements des populations de $G$. tachinoides observés au cours de l'année.

L'examen de ce tableau et de la figure 2 montre qu'en période de très basses eaux, comme ce fut le cas en 1973, une quatrième zone végétale constituée surtout de Mimosa pigra ou parfois de Salix ledermanni est mise à découvert et constitue les seuls lieux de survie des glossines. Celles-ci abandonnent totalement les gîtes habituels devenus trop arides où l'allongement de la période de dessèchement a déterminé la mort de nombreux arbres: Morelia notamment et même Mimosées. Grou- 


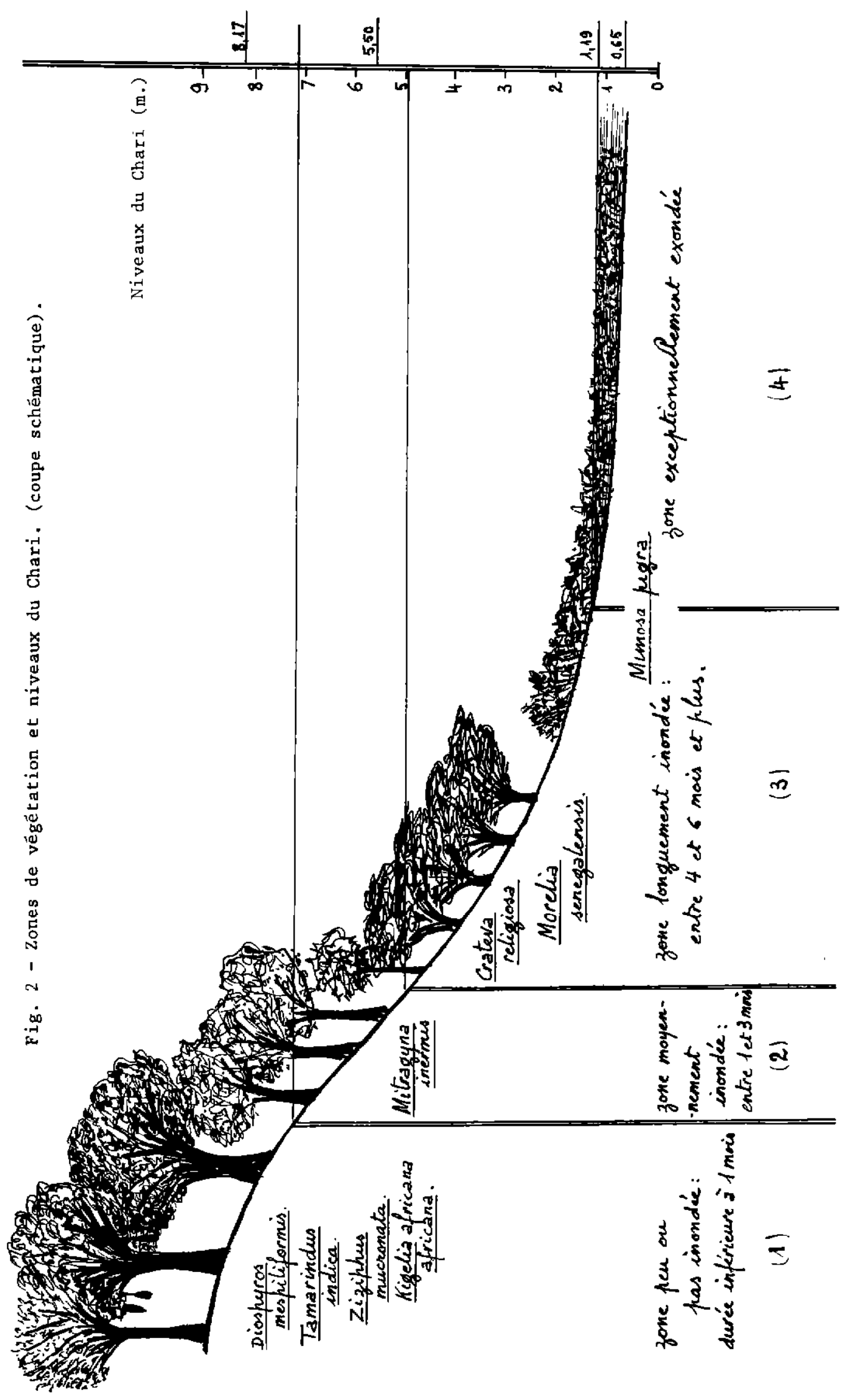


TABLEAU N"II.-Zones vêgêtales occupêtes par les glossines selon les mois

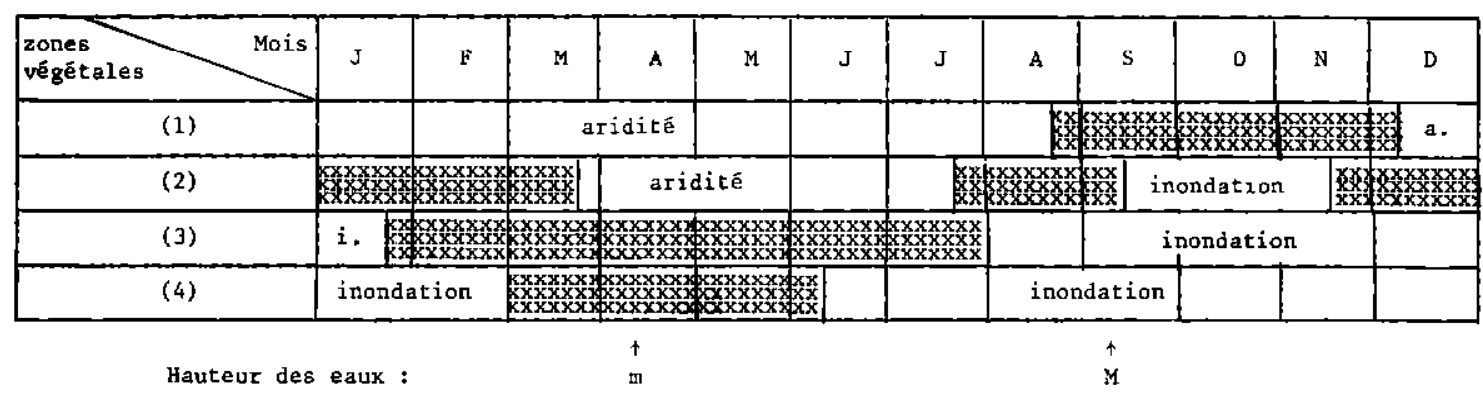

(Prêsence de glossines XXXX ; Absence de glossines : par aridité ou par inondation.

pées dans ces bas-fonds touffus, les glossines femelles peuvent déposer leur larve, mais pour l'ensemble de la population des tsé-tsé, la recherche de nourriture pose un problème qui ne peut être résolu que par celles proches des endroits où le gibier se rassemble, près des nappes d'eau où il a l'occasion de s'abreuver. Il est probable que, pendant cette période critique, un grand nombre de glossines meurent d'inanition.

A la remontée des eaux, les mouches adultes, survivantes ou écloses de pupes formées sous les mimosées, trouvent à grand peine refuge dans la végétation voisine constituée par les gîtes traditionnels souvent éclaircis par la sécheresse et qui protègent mal des rigueurs climatiques. Les pupes, non encore écloses sous les mimosées, sont alors noyées.

Il faut souligner également l'action " adjuvante " des habitants qui défrichent, déboisent tout ou partie de certaines zones (2) et (3) pour y pratiquer des cultures de décrues. Ces interventions enlèvent toute possibilité de survie aux glossines évacuant les mimosées voisines. Cette action de l'homme tend à se généraliser tout au long du Chari et du Logone (4) et contribue dans une large mesure à réduire et à morceler la distribution de $G$. tachinoides. Les prospections menées récemment, aux hautes eaux et dès le début de la décrue ont confirmé l'absence de glossines en des lieux, non traités par les insecticides, où elles étaient normalement abondantes à la même époque : grands gîtes de Dougia, de Mani, etc.

La destruction progressive des gîtes à $G$. tachinoides, amorcée depuis longtemps par l'intervention des habitants, se trouve accélérée depuis 1972 par action de la sécheresse et sera encore nettement marquée pendant la prochaine saison chaude, en 1974.

\section{SECHERESSE ET LUTTE CONTRE G. TACHINOIDES}

L'exécution du programme de lutte contre $G$. tachinoides dans la vallée du bas-Chari, établi dès 1966, coïncide avec l'installation de cette période aiguë de sécheresse.

Le principe de lutte adopté consiste à appliquer sélectivement, sur les lieux de repos recherchés par les glossines, un insecticide rémanent : le D.D.T. en solution à 2,5 p. 100 .

Les études écologiques préalables avaient permis de préciser la nature végétale et l'étendue de ces lieux de repos, variables selon les périodes de l'année, et d'en déduire une limitation rationnelle des surfaces à pulvériser. Nos observations avaient montré également que l'effet insecticide du D.D.T. appliqué sur les écorces se prolongeait au-delà de 3 mois. Il convenait de limiter les pulvérisations :

- à la période chaude de l'année lorsque les glossines sont à leur maximum de concentration;

- aux seuls lieux de repos fréquentés à cette époque: bordures de Mimosées, parties inférieures à $1 \mathrm{~m}$ des troncs de Morelia et des Mitragyna, en limite de ces derniers.

L'installation de la sécheresse, dont les effets locaux ont été décrits précédemment, modifie nettement ces principes applicables en année normale. Le dessèchement des gîtes de bordure à Morelia et la mise à nu de vastes étendues de mimosées, en imposant aux glossines des habitats inhabituels, entraînent d'une part une forte diminution voire une suppression des pulvérisations sur la végétation arborée et, d'autre part, une augmentation des interventions au niveau des fourrés à Mimosées dont la surface est devenue immense. La pénétra- 
tion en est difficile et la vitesse linéaire de progression des équipes de travail se trouve considérablement ralentie. De plus, la consommation d'insecticide y est nettement augmentée.

Mais il convient de remarquer qu'une sélection de ces champs de Mimosées s'impose:

- certains d'entre eux sont complètement isolés, loin de toute végétation arborée dense et de tous points d'eau voisins. Aucun gibier ne s'y rencontre et les glossines sont naturellement absentes de ces endroits. Il devient alors inutile de les traiter car, rapidement inondés, ils ne seront jamais habités par les glossines.

- d'autres, possédant encore quelques basfonds humides et situés à proximité des lieux de rassemblement des animaux peuvent retenir quelques populations de glossines. Exondés peu longtemps, l'intervention n'y a pour effet que de détruire les seules mouches présentes; la montée des eaux qui suivra rend, en noyant ces endroits, l'emploi d'un insecticide rémanent tout à fait superflu. De plus, l'assèchement de la végétation voisine suffit à s'opposer à l'installation des rares glossines ayant échappé au traitement. Il apparaît alors possible, en année de sécheresse exceptionnelle, de ne traiter qu'au moment des plus basses eaux et de n'utiliser que des insecticides aux effets fugaces n'entraînant pas les conséquences lointaines, polluantes, néfastes pour la faune sauvage environnante, trop souvent à déplorer à la suite d'applications massives d'insecticides chlorés.

La distinction qui vient d'être faite entraîne la nécessité de faire un choix dans les lieux susceptibles d'être traités, si l'on veut conserver les avantages de la sélectivité.

- En année normale, les prospections préalables aux pulvérisations peuvent être menées rapidement, ne considérant que l'aspect général des gites traditionnels et les attaques relativement fréquentes des mouches encore nombreuses.

- En année anormale de sécheresse, la sélection des gîtes à traiter est plus délicate. Elle doit faire intervenir l'examen de tous les facteurs écologiques contribuant au maintien des glossines dans une zone végétale donnée : densité de la végétation, humidité sous ombrage, proximité des points d'eau et fréquence du gibier. De plus, les tsé-tsé ne manifestent leur présence qu'aux heures où les conditions climatiques déterminent leur maximum d'activité : mi-journée en saison fraîche, matin et soir en saison chaude. Ce point est extrêmement important à considérer avant de pouvoir conclure à l'absence ou à la présence de glossines. En période sèche où les durées d'activité sont limitées, toute recherche hors des heures favorables peut entraîner des conclusions fausses.

En année de sécheresse, le traitement des gîtes, sélectionnés par ces prospections préalables, pourra être fait tardivement, en saison chaude, lorsque les mouches sont à leur maximum de concentration; la durée d'intervention sera ainsi réduite.

Les pulvérisations des massifs de mimosées entraînent cependant une préparation préalable du terrain pour en faciliter les accès en profondeur. Toutefois, il apparaît inutile de poursuivre le traitement vers l'intérieur de ces massifs au-delà d'une dizaine de mètres, les glossines ne pénétrant la végétation que par ses limites dégagées. L'application de l'insecticide pourra être étendue aux troncs des arbres voisins appartenant à des gîtes de bordure encore denses, peu affectés par la dessiccation.

\section{CONCLUSION}

L'insuffisance et la mauvaise répartition des pluies, localement et sur les hautes vallées du réseau hydrographique du Chari, déterminent en aval de N'Djamena un flétrissement précoce de la végétation riveraine. Celui-ci est particulièrement accentué le long des défluents et des mares intérieures qui n'ont pas été normalement alimentés en eau lors des crues du Chari dont le niveau s'est révélé dès 1972, particulièrement bas. Les minimums de saison chaude sont devenus en conséquence très inférieurs à la normale, voire nuls, dans quelques cours d'eau qui ont été pendant plusieurs semaines totalement asséchés. $\mathrm{La}$ végétation bordante s'est montrée rapidement inhabitable pour les glossines qui ne peuvent alors être rencontrées que dans les denses massifs de Mimosées occupant les bas-fonds conservant encore quelque humidité. Leur survie n'y reste cependant possible que dans la mesure où la faune sauvage peut se maintenir à proximité, bénéficiant de rares points d'eau pour s'abreuver. 
Dans de telles conditions, l'exécution d'un programme de lutte apparaît en grande partie facilité; pouvant être limitée dans le temps à la période de concentration maximale des glossines et géographiquement aux seuls endroits où elles se réfugient. Mais, dans une certaine mesure, la préparation des pulvérisations au niveau des champs de mimosées peut parfois entraîner un ralentissement dans l'exécution du travail qui peut cependant être estimé lors des prospections.

Il convient, par ailleurs, de souligner l'action destructrice des habitants sur les galeries ripicoles dont les effets s'ajoutent aux précédents pour réduire la distribution de $G$. tachinoides.

\section{SUMMARY}

\section{Drought, ecology, and control of Glossina tachinoides in the Lower Chari area}

The severe drought which has become prevailing in the Sahelian zone of Africa for some years has an effect at the level of the riparian galleries in the Chari Valley. It significantly modifies:

- the ecological conditions for $G$. tachinoides which sees its populations decrease in number by limiting their presence only to the few sites free of drought;

- the conditions of insecticide application in the course of the implementation of the control campaign carried out at present downstream of N'Djamena.

\section{RESUMEN}

\section{Sequía, ecologia y lucha por insecticidas contra Glossina tachinoides en la región del bajo-Chari}

La sequía importante que ocurre en Africa saheliana desde algunos años ejerce su influencia en la vegetatión de las orillas del Chari.

Modifica: las condiciones ecologicas para con $G$. tachinoides cuyas poblaciones siguen reduciendo y no se encuentran más que en sitios no secos; las condiciones de aplicación del insecticida durante la ejecución de la campaña de lucha actualmente efectuada más abajo de N'Djamena.

\section{BIBLIOGRAPHIE}

1. CUISANCE (D.). Evolution, sous l'action de la sécheresse, d'une population isolée de $G$. tachinoides West.; région du Bas-Logone. (Sous presse).

2. GRUVEL (J.). Les glossines, vectrices des trypanosomiases au Tchad. Rev. Elev, Méd. vét. Pays trop., 1966, 19 (2): 169-211.

3. GRUVEL (J.), TRONCY (P.M.) et TIBAYRENC (R.). Contribution à la connaissance de la distribution des glossines au Nord Cameroun. Rev. Elev. Méd. vét. Pays trop., 1970, 23 (1): 83-91.

4. GRUVEL (J.). Contribution à l'étude écologique de $G$. tachinoides West., dans la réserve de Kalamaloué, vallée du Bas-Chari. Thèse Doct. Etat Sci. nat. Paris. 1974.

5. Mac LENNAN (K. J. R.). Fifty years of entomological research in relation to glossina extermination: current progress and future trends in Northern Nigeria. Proc. ent. Soc. Nig., 1968, 1 : 83-87.

6. Mac LENNAN (K. J. R.). A consideration of environmental consequences following anti-tsetse operations in Nigeria. Trop. anim. Hith. Prod., $1973,5: 40-45$. 